\title{
Molecular dynamics simulations of oxide memristors: thermal effects
}

\author{
S.E. Savel'ev • A.S. Alexandrov • A.M. Bratkovsky • \\ R. Stanley Williams
}

Received: 1 December 2010 / Accepted: 22 December 2010 / Published online: 5 February 2011

(C) The Author(s) 2011. This article is published with open access at Springerlink.com

\begin{abstract}
We have extended our recent molecular-dynamic simulations of memristors to include the effect of thermal inhomogeneities on mobile ionic species appearing during operation of the device. Simulations show a competition between an attractive short-ranged interaction between oxygen vacancies and an enhanced local temperature in creating/destroying the conducting oxygen channels. Such a competition would strongly affect the performance of the memristive devices.
\end{abstract}

There are many challenges in understanding and controlling the coupled electronic and ionic kinetic phenomena dominating the behavior of oxide switching devices like $\mathrm{Pt} / \mathrm{TiO}_{2} / \mathrm{Pt}$, which is an exemplar memristor (resistor with memory) [1], a fourth passive circuit element originally postulated by Leon Chua in 1971 [2]. It has been demonstrated unambiguously that bipolar switching involves changes to the electronic barrier at the $\mathrm{Pt} / \mathrm{TiO}_{2}$ interface due to the drift of positively charged oxygen vacancies under an applied electric field [1]. Various direct measurements revealed formation of localized conducting channels in $\mathrm{TiO}_{2}$ : pressure modulated conductance microscopy [3, 4], conducting atomic force microscopy (AFM) [5], scanning transmission $\mathrm{X}$-ray microscopy [6], and in-situ transmission electron microscopy [7]. On the basis of these measurements, it became quite clear that the vacancy drift towards the interface

S.E. Savel'ev · A.S. Alexandrov

Department of Physics, Loughborough University, Loughborough LE11 3TU, UK

A.S. Alexandrov · A.M. Bratkovsky $(\bowtie) \cdot$ R.S. Williams Hewlett-Packard Laboratories, 1501 Page Mill Road, Palo Alto, CA 94304, USA

e-mail: alex.bratkovski@hp.com creates conducting channels that shunt, or short-circuit, the electronic barrier and switch the device ON [1]. The drift of vacancies away from the interface breaks such channels, recovering the electronic barrier to switch the junction OFF.

The microscopic understanding of the atomic-scale mechanism and identification of the material changes within an insulating barrier appear to be invaluable for controlling and improving the memristor performance. The number of oxygen vacancies within the volume $10 \times 10 \times 2 \mathrm{~nm}^{3}$ of perspective nano-memristors could be as small as a thousand, so that the conventional statistical (diffusion) approach for dealing with many-particle systems might fail. However, the dynamic phase transitions as well as the competition between thermal fluctuations and particle-particle interactions in stochastic transport can be investigated with the use of Molecular Dynamics (MD) simulations of the Langevin equations describing the thermal diffusion and drift of individual interacting particles [8].

Recently, we have proposed a model for the kinetic behavior of oxide memristors and simulated its toy analog using the MD [9]. Our MD simulations revealed a significant departure of the vacancy distributions across the device from that expected within a standard drift-diffusion approximation. Another important step would be to include thermal effects via local changes in the diffusion coefficient. Indeed, the channel formation in systems like $\mathrm{TiO}_{2}, \mathrm{NiO}$, $\mathrm{VO}_{2}$ is certainly accompanied by local heating that is witnessed by the local emergence of high-temperature phases and is observed by thermal microscopy. For instance, fresh $\mathrm{TiO}_{2}$ samples have an amorphous layer of titanium dioxide. To make the device switch in a repeatable manner, a forming step of rather large voltage pulse is usually required to create a vacancy-rich region (forming is not required if e.g. a vacancy-rich layer is provided intentionally by fabrication). During this process, the $\mathrm{TiO}_{2}$ anatase phase forms near 


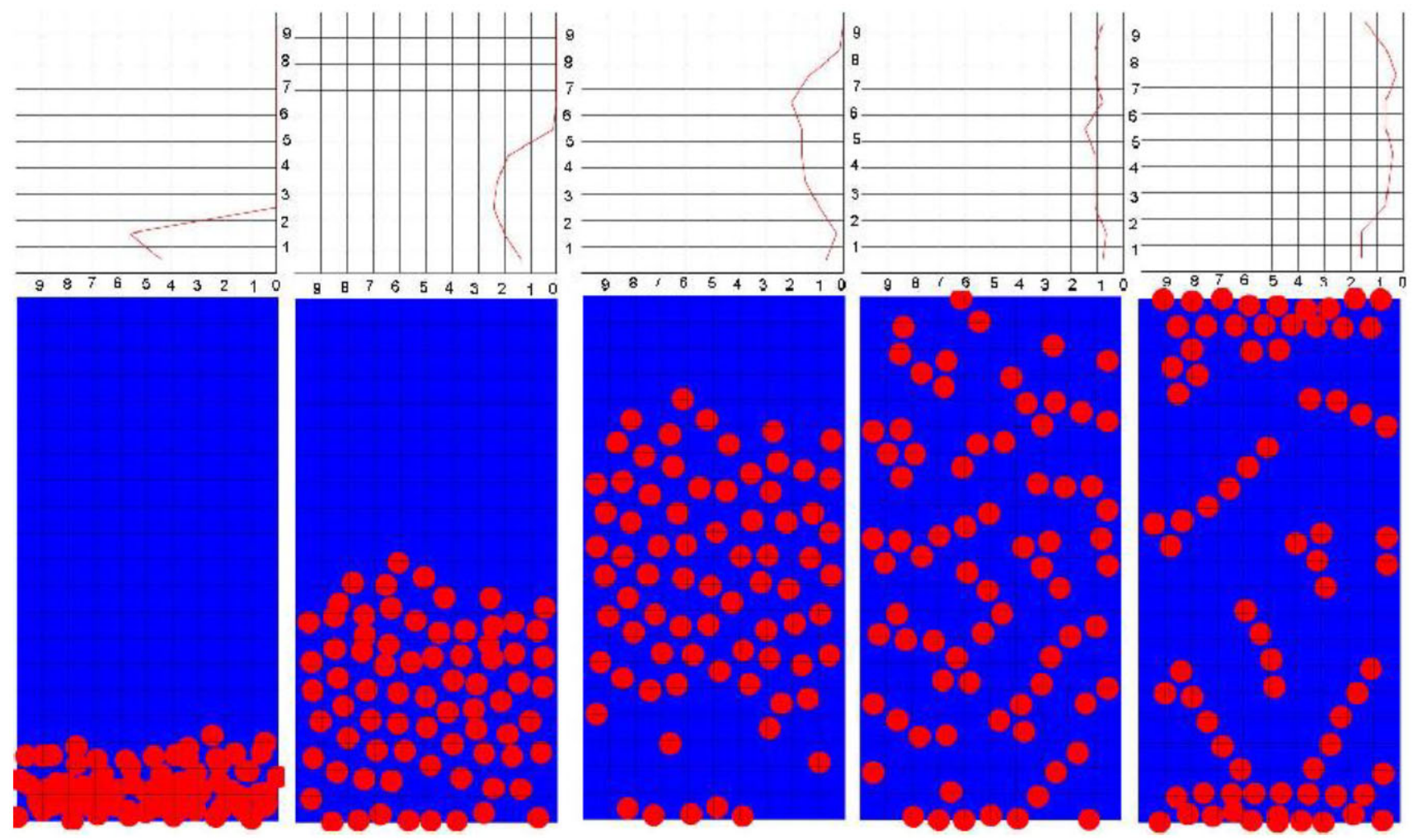

Fig. 1 Simulation of dynamics of vacancies during and after the external voltage pulse is applied. Parameters are the same as in Fig. 1 in Ref. [9]. Top row shows the distribution of vacancies $n(y)$ across the sample (with $y$-axes aligned with those in the bottom row.) One can observe how the vacancies redistribute at various simulation times ( $t=0,0.5,0.9,1.5,5$ left to right, the time measured in the pulse

the conducting channel, which nominally requires temperatures over $350^{\circ} \mathrm{C}$ [6]. Studying local thermal effects during switching in $\mathrm{TiO}_{2}$ provides strong evidence for local heating [10]. Unambiguous evidence for local heating accompanying conducting channel formation comes from third harmonic generation [11]; low resistance states (LRS) in $\mathrm{NiO}$ showing unipolar switching were strongly nonlinear with variations in the resistance as large as $60 \%$, which was most likely caused by the Joule heating of conducting channels inside the films.

Here, we extend our MD of oxygen vacancies interacting via realistic potentials and driven by an external bias voltage by taking into account temperature gradients in thin films of oxide memristors. Based on the observations of oxygen vacancy migration and clustering in bulk [12-17] and nanoscale $[6,7]$ samples of $\mathrm{TiO}_{2}$ induced by an electric field, we have modeled a memristor [9] as shown in Fig. 1. In our model, there is a reduced rutile thin layer $\mathrm{TiO}_{2-x}$ near one of the metallic electrodes stabilized by the Coulomb mirror potential. Vacancies from this layer can drift toward the opposite electrode in an electric field. Vacancies interact with each other via the pairwise potential $W$, and with exter- duration interval). The final configuration (last column, $t=5$ ) is a practically stable distribution with a larger density of vacancies near the top and the bottom electrodes due to the attractive interaction with the image charges. These patterns are slightly different from Fig. 1 in Ref. [9] since we have many metastable states that may trap the vacancies

nal fields corresponding to the time-dependent deterministic force $F$. The environment exerts an independent Gaussian random force, $\vec{\xi}$, on each particle with zero mean and intensity controlled by the temperature. In the overdamped regime (where inertia is negligible compared to the viscous damping), the Langevin equation describing the driftdiffusion of the $i$ th particle is [8]

$$
\begin{aligned}
\eta \frac{d x_{i}^{\alpha}}{d t}= & F_{i}^{\alpha}\left(\boldsymbol{x}_{i}, t\right)-\sum_{j \neq i} \frac{\partial W\left(\boldsymbol{x}_{i}-\boldsymbol{x}_{j}\right)}{\partial x_{i}^{\alpha}} \\
& +\sqrt{2 k_{B} T \eta} \gamma^{\alpha} \xi_{i}^{\alpha}(t) .
\end{aligned}
$$

where $x_{i}^{\alpha}$ is the $\alpha$-coordinate of the $i$ th vacancy, $\eta$ is the friction coefficient, $F_{i}^{\alpha}$ is the $\alpha$-component of electric pulse force, $k_{B}$ is the Boltzmann constant, $\gamma$ is related to diffusion anisotropy, and $\xi^{i}$ is a random force.

The $\mathrm{O}^{2-}$ vacancy-vacancy interaction potential, which is crucial for their clustering and phase transformations, can be modeled as

$W(R)=A e^{-R / a}-\frac{B}{R^{6}}+\frac{e^{2}}{\pi \epsilon_{0} \epsilon R}$, 


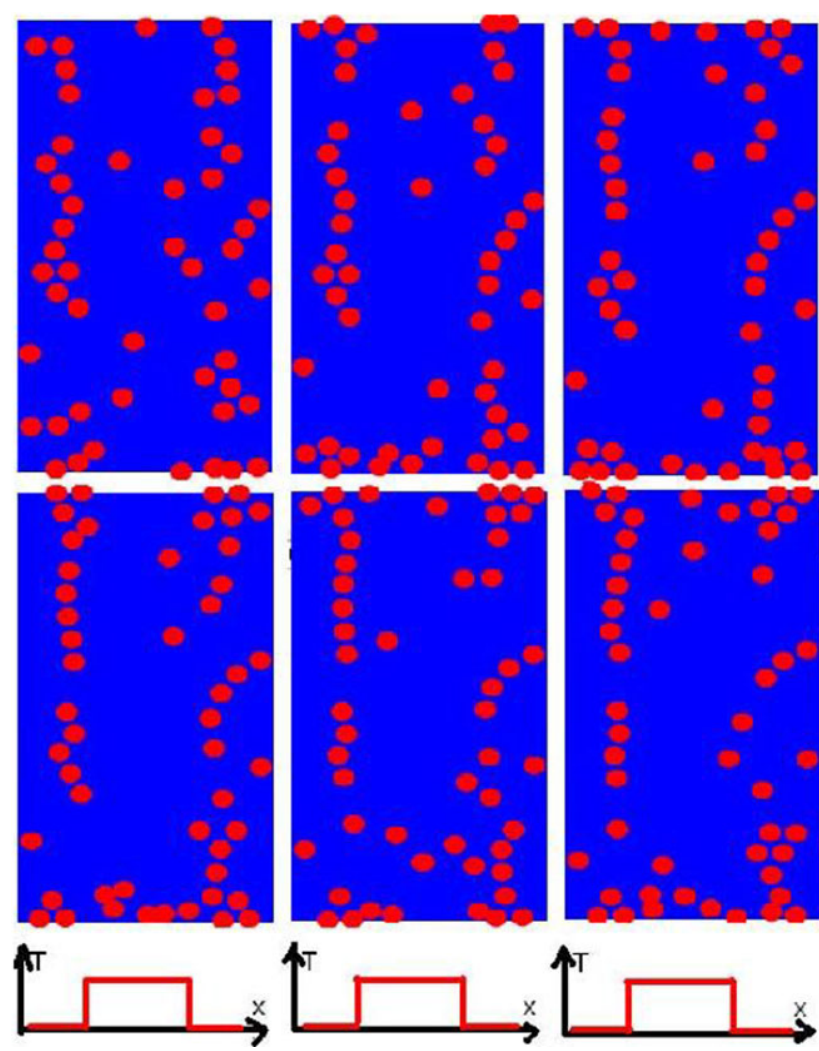

Fig. 2 Vacancy distribution formed long time after the voltage pulse has been applied (top panel: $t=5,5.1,5.5$; bottom panel: $t=5.7,6$, $6.5)$ where the time is measured in the pulse duration interval. All parameters are the same as in Fig. 1 apart from the anisotropy coefficient $\gamma=1 / 3$. Temperature varies across the sample with the amplitude $T_{0}$ equal to the depth of potential minima corresponding to the force (3). This temperature is enough to break up the vacancy chains

where the short-range repulsive and attractive parts are represented with the parameters $A=22764.0 \mathrm{eV}, a=0.01490$ $\mathrm{nm}$, and $B=27.88 \times 10^{-6} \mathrm{eV} \mathrm{nm} \mathrm{[18],} \mathrm{and} \mathrm{the} \mathrm{long-range}$ Coulomb repulsion is given by the last term. More generally, the interaction parameters $A, B, a$ vary from one oxide to another, and the dielectric constant $\epsilon$ may also vary from sample to sample.

In contrast with strongly inhomogeneous (internal) electric fields, we have assumed in Ref. [9] that the diffusion coefficient is homogeneous in the simulated region, which corresponds to a homogeneous temperature distributions. Here, we extend our model by taking into account local overheating in the sample, which appears to have a drastic effect on the vacancy distribution pattern. Indeed, the diffusion coefficient exponentially depends on temperature [19], so that the variations of the local temperature during switching could significantly affect the formation and disintegration of conducting channels.

We have performed the MD simulations of a toy memristor with relatively small number of vacancies, $N=75$ (Fig. 1). Initially, we have placed all the vacancies ran- domly near the bottom of a sample (see Fig. 1 for the initial distribution) and then let these vacancies evolve according to (1) inside a rectangular box that mimics the actual device. We use the $2 \mathrm{D}$ simulation area with the aspect ratio $L_{y} / L_{x}=2$ and periodic boundary conditions (BC) along the $x$-direction. Note that the periodic BC allow us to simulate an infinite area sample using a rather small number of particles. To use periodic BC, we include periodic images of the vacancies with respect to vertical boundaries of the simulation box. We also incorporate opposite polarity charges by adding mirror images of vacancies with respect to the top and the bottom of the sample.

Since we simulate a limited number of vacancies, one can refer to each particle in our simulations as a cluster of vacancies, where a cluster-cluster interaction may be described by the combination of the Lennard-Jones and Coulomb potentials acting with the force

$F(r)=\frac{1}{r}\left\{12 E_{\mathrm{LJ}}\left[\left(\frac{r_{\mathrm{min}}}{r}\right)^{12}-\left(\frac{r_{\mathrm{min}}}{r}\right)^{6}\right]+E_{c} \frac{r_{\min }}{r}\right\}$,

where the relative strength of the Coulomb potential is given by $E_{c} / E_{\mathrm{LJ}}=2$. This results in the position of the potential maxima $r_{\max } \approx 2 r_{\min }$ and the height of the potential barrier on the order of the depth of the potential well. The pulse strength is taken to be about ten times stronger than the maximum attractive force between vacancy clusters.

We present the results of simulation for the device with homogeneous temperature distribution [9] in Fig. 1. Different columns refer to different moments in time and the top row shows the corresponding local densities. The first column shows the initial distribution of vacancies before simulations: all of them are located at the bottom of the sample. The second and third columns show distribution in the middle and at the end of the pulse. One can see that vacancies are pushed by the pulse to the center of the sample: the density distribution is spread out and its maximum moves towards the top of the sample with a constant velocity. Moreover, even after switching the pulse off the vacancy distribution front keeps moving towards the top of the sample while the vacancies gradually form filamentary clusters (fourth column). Note that, obviously, such filamentary clusters are impossible to reveal when modeling vacancy dynamics by using just vacancy density $n(y)$. Indeed, one cannot see anything interesting from the average density profile (top panel of the fourth column). Finally, vacancies form the percolation paths that are stable during all simulations while the average density again shows nothing interesting apart from a trivial fact that $n(y)$ is slightly larger at the top and the bottom of the sample due to the vacancies being attracted to their image charges.

Comparison of our simulations with the averaged density description indicates the following: (i) modeling vacancy dynamics by using macroscopic distributions fails to 


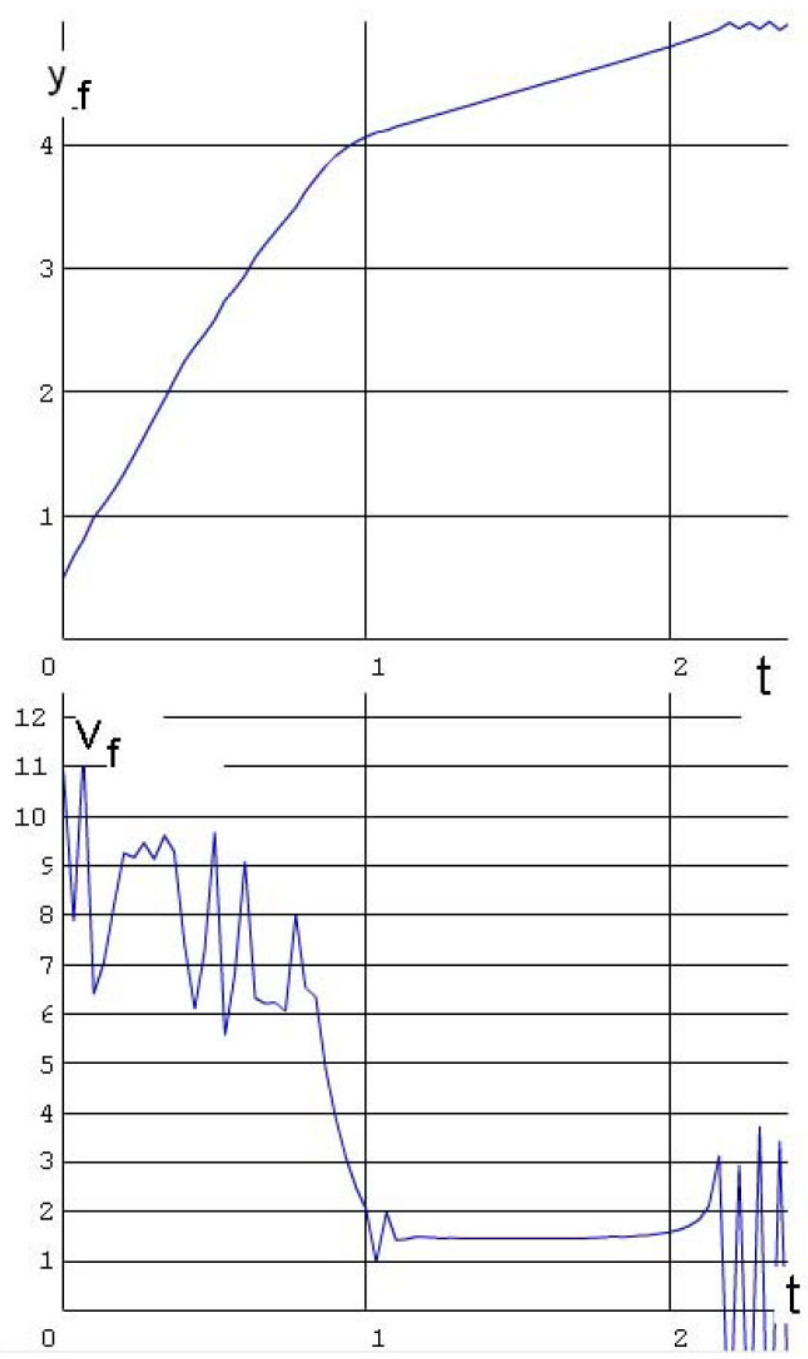

Fig. 3 The location $y_{f}$ and the velocity $v_{f}$ of the front of vacancy distribution versus time. One can see two different regimes of vacancy propagation through the sample: pulse driven at time $t<1$ and free expansion due to the Coulomb repulsion between the charged vacancies $(t>1)$ ending by establishing a stable distribution due to vacancy confinement between sample boundaries. In both cases expansion is linear in time meaning that the diffusion driven expansion $\propto \sqrt{t}$ is insignificant

Fig. 4 The same as in Fig. 2 for time $(t=5 ; 5.5 ; 6)$, but now with a temperature gradient along the sample ( $y$-axis)

$T=T_{0}(5 y-4 h) \Theta(y-4 h / 5) / h$ and $T_{0}$ is five times larger than the potential minimum depth. No stable configuration is formed in the hot top part of the sample. The temperature profile $T=T(y)$ is shown in the right sidebar describe filamentary cluster formation, (ii) there are plenty of metastable vacancy distributions and, thus, one can expect formation of slightly different percolation paths even for a series of experiments on the same sample, (iii) MD simulations are ideal to describe the statistics of such percolation path formation. Further deviation from the standard diffusion approach can be seen when monitoring motion of vacancy distribution front $y_{f}=\max _{i}\left(y_{i}\right)$ (see Fig. 3), where the maximum is taken with respect to all the vacancies. One can see two different expansion regimes for the front: a pulse driven regime and another one driven by the Coulomb repulsion between the vacancies. Note that the velocity of expansion is always constant indicating that diffusion driven expansion is negligible in our system.

The temperature gradients can change the vacancy distribution patterns significantly. As examples, we have considered variations of the temperature parallel to the electrodes, $T(x)$, Fig. 2, and across $T(y)$, Fig. 4, our toy memristor. These gradients can be due to, e.g., spatial variations of cooling or applying the heat sinks to the sample. We use a square temperature profile along the film $T(x)=T_{0} \Theta(d / 4-|x|)$ in Fig. 2, where $x=0$ corresponds to the middle of the sample, $d$ is the width of this region, and $\Theta$ is the step function. This mimics possible temperature oscillations along the sample footprint. In the present study, we assume a temperature independent mobility [i.e. a constant $\eta$ in (1)], which would formally correspond to quantum tunneling rather than thermally activated hopping. One can see that even in this regime the vacancies tend to escape from the hot regions, resulting in a more pronounced formation of percolation vacancy channels in cold regions of the simulated sample.

A somewhat similar situation occurs if we assume that the top of the sample is hot with respect to the rest of the sample, i.e., $T(y)=T_{0}\left(y-y_{0}\right) \Theta\left(y-y_{0}\right) /\left(h-y_{0}\right)$. In other words, we assume that the sample is cold at the bottom ( $T=0$ for $\left.y<y_{0}\right)$ and the temperature linearly increases in the region $y_{0}<y<h$, where $h$ is the sample thickness. The simulation results for such a distribution of temperature are shown in Fig. 4. As one can see, vacancies cannot form
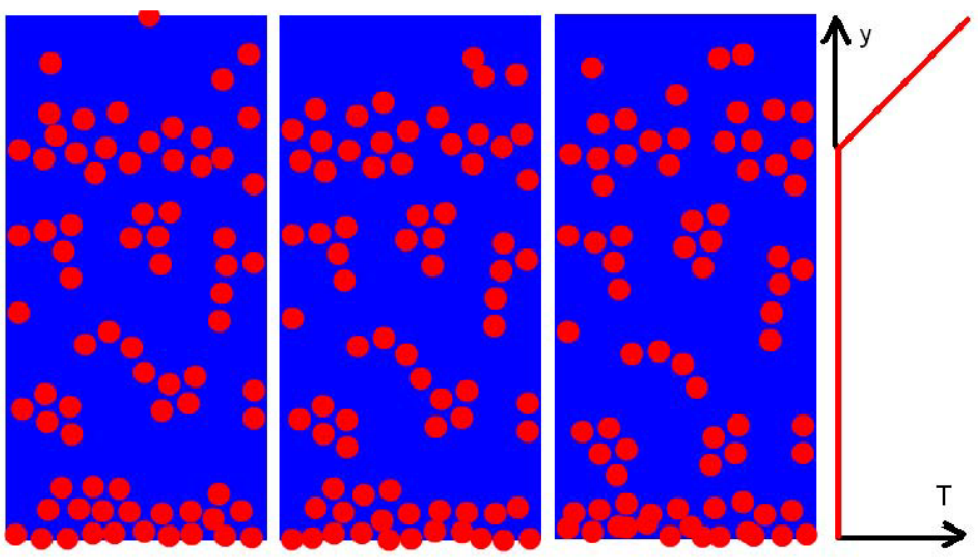
stable configurations in the hot region near the top of the sample: only limited number of vacancies can enter the hot region, these vacancies quickly wander through this region and return back into the cold part of the sample. In contrast, the vacancy distribution is quite stable in the bottom cold region of the sample, as expected. This behavior may be relevant for the observed 'inversion' of vacancy distribution in Ref. [20].

One can clearly see in Figs. 2 and 4 that even a moderate temperature/diffusion gradient drastically affects the formation of percolation paths. To be compared to the homogeneous temperature regime, Fig. 1, the vacancies are escaping hot regions of the sample and aggregating into the chain formations in the cold regions. This suggests a strong competition between the local heating and the attractive part of the vacancy-vacancy interaction resulting in disintegration/creation of the conducting vacancy channels. Note that we do not consider interactions of vacancies with the crystal matrix, which can be incorporated by adding a crystal potential $U_{\mathrm{cr}}(x, y)$ in our Langevin equations. If potential barriers of $U_{\mathrm{cr}}(x, y)$ are high enough and the motion of vacancies occurs only via thermal hopping between nearest potential minima of $U_{\mathrm{cr}}(x, y)$, then an exponential temperature dependence of the effective mobility of vacancies can be observed. Thus, the competition between the local heating and vacancy interactions should be much stronger in the thermally activated (hopping) regime, when the effective mobility (proportional to $\eta$ ) and the effective diffusion coefficient exponentially depend on temperature [21].

In conclusion, we have demonstrated that temperature gradients across memristive devices, producing strong variations of the diffusion rate, affect the vacancy patterns in memristors quite dramatically. Our simulations indicate that variations of temperature across the memristor favor the formation of short-circuiting or shunting vacancy channels. At the same time, considerable temperature gradients in the growth direction, in contrast, produce vacancy-poor regions where vacancy shunts are not formed and electron percolation paths are blocked. Further studies of these effects may have profound impact on present understanding of physics of memristive devices.
Open Access This article is distributed under the terms of the Creative Commons Attribution Noncommercial License which permits any noncommercial use, distribution, and reproduction in any medium, provided the original author(s) and source are credited.

\section{References}

1. J.J. Yang, M.D. Pickett, X.M. Li et al., Nat. Nanotechnol. 3, 429 (2008)

2. D.B. Strukov, G.S. Snider, D.R. Stewart, R.S. Williams, Nature 453, 80 (2008)

3. J.J. Yang, F. Miao, M.D. Pickett, D.A.A. Ohlberg, D.R. Stewart, C.N. Lau, R.S. Williams, Nanotechnology 20, 215201 (2009)

4. F. Miao, J.J. Yang, J.P. Strachan, D. Stewart, R.S. Williams, C.N. Lau, Appl. Phys. Lett. 95, 113503 (2009)

5. R. Mustermann, J. Yang, J.P. Strachan, G. Medeiros-Ribeiro, R. Dittmann, R. Waser, Phys. Status Solidi 4, 16 (2009)

6. J.P. Strachan, M.D. Pickett, J.J. Yang, S. Aloni, A.L.D. Kilcoyne, G. Medeiros-Ribeiro, R.S. Williams, Adv. Mater. 22, $3573(2010)$

7. D.-H. Kwon, K.M. Kim, J.H. Jang, J.M. Jeon, M.H. Lee, G.H. Kim, X.-S. Li, G.-S. Park, B. Lee, S. Han, M. Kim, C.S. Hwang, Nat. Nanotechnol. 5, 148 (2010)

8. S. Savel'ev, F. Marchesoni, F. Nori, Phys. Rev. E 70, 061107 (2004), and references therein

9. S.E. Savel'ev, A.S. Alexandrov, A.M. Bratkovsky, R. Stanley Williams, arXiv: 1010.5656

10. J. Borghetti, D.B. Strukov, M.D. Pickett, J. Yang, D.R. Stewart, R.S. Williams, J. Appl. Phys. 106, 124504 (2009)

11. S.B. Lee, S.C. Chae, S.H. Chang, J.S. Lee, S. Park, Y. Jo, S. Seo, B. Kahng, T.W. Noh, Appl. Phys. Lett. 93, 252102 (2008)

12. H. Miyaoka, G. Mizutani, H. Sano, M. Omote, K. Nakatsuji, F. Komori, Solid State Commun. 123, 399 (2002)

13. K. Tsunoda et al., Appl. Phys. Lett. 90, 113501 (2007)

14. K.M. Kim et al., Appl. Phys. Lett. 90, 242906 (2007)

15. J.R. Jameson et al., Appl. Phys. Lett. 91, 112101 (2007)

16. H. Shima et al., Appl. Phys. Lett. 94, 082905 (2009)

17. Ni Zhong et al., Appl. Phys. Lett. 96, 042107 (2010); and references therein

18. C. Meis, J.L. Fleche, Solid State Ion. 101, 333 (1997)

19. M. Radecka, P. Sobas, M. Rekas, Solid State Ion. 119, 55 (1999)

20. J.J. Yang, J. Borghetti, D. Murphy, D.R. Stewart, R.S. Williams, Adv. Mater. 21, 3754 (2009)

21. S.E. Savel'ev, A.S. Alexandrov, A.M. Bratkovsky, R. Stanley Williams (in preparation) 